\title{
Magnetism in C- or N-doped MgO and ZnO: A Density-Functional Study of Impurity Pairs
}

\author{
Hua Wu, ${ }^{1,2, *}$ Alessandro Stroppa,${ }^{3}$ Sung Sakong, ${ }^{4}$ Silvia Picozzi, ${ }^{3}$ Matthias Scheffler, ${ }^{5}$ and Peter Kratzer ${ }^{4}$ \\ ${ }^{1}$ II. Physikalisches Institut, Universität zu Köln, Zülpicher Str. 77, 50937 Köln, Germany \\ ${ }^{2}$ Department of Physics, Fudan University, Shanghai 200433, China \\ ${ }^{3}$ CNR-SPIN, L'Aquila, Italy \\ ${ }^{4}$ Fakultät für Physik and Center for Nanointegration (CeNIDE), Universität Duisburg-Essen, \\ Lotharstr. 1, 47048 Duisburg, Germany \\ ${ }^{5}$ Fritz-Haber-Institut der Max-Planck-Gesellschaft, Faradayweg 4-6, 14195 Berlin, Germany
}

(Received 15 March 2010; published 27 December 2010)

\begin{abstract}
It is shown that substitution of $\mathrm{C}$ or $\mathrm{N}$ for $\mathrm{O}$ recently proposed as a way to create ferromagnetism in otherwise nonmagnetic oxide insulators is curtailed by formation of impurity pairs, and the resultant $\mathrm{C}_{2}$ spin $=1$ dimers as well as the isoelectronic $\mathrm{N}_{2}^{2+}$ interact antiferromagnetically in $p$-type $\mathrm{MgO}$. For $\mathrm{C}$-doped $\mathrm{ZnO}$, however, we demonstrate using the Heyd-Scuseria-Ernzerhof hybrid functional that a resonance of the spin-polarized $\mathrm{C}_{2} p p \pi^{*}$ states with the host conduction band results in a long-range ferromagnetic interaction. Magnetism of open-shell impurity molecules is proposed as a possible route to $d^{0}$-ferromagnetism in oxide spintronic materials.
\end{abstract}

DOI: 10.1103/PhysRevLett.105.267203

PACS numbers: 75.50.Pp, 71.20.-b, 71.70.-d, 71.15.Mb

Ferromagnetic (FM) semiconductors or insulators that are free of transition-metal or rare-earth species are nowadays perceived as a class of systems displaying $d^{0}$ magnetism [1-3]. As a common feature, defects with $s p$-type orbital character carry the magnetic moment in these systems. One widely discussed scenario for functionalizing oxides as $d^{0}$ dilute magnetic semiconductors involves the magnetic polarization of valence states by the substitution of oxygen with $s p$-type impurities of lower valency. To maximize the Hund exchange at the impurity sites, doping by the $2 p$ elements $\mathrm{C}$ or $\mathrm{N}$ appears most promising. For example, local-density approximation (LDA) calculations predicted that group-II oxides $M \mathrm{O}(M=\mathrm{Mg}, \mathrm{Ca}, \mathrm{Sr}, \mathrm{Ba})$ become FM when doped with $\mathrm{C}$ or $\mathrm{N}$ [4,5]. Moreover, experiments and computations demonstrated that C-doped $\mathrm{ZnO}$ is FM even above room temperature [6-9]. Typically samples with a very high concentration of impurities are used. Under these conditions, it was believed that the FM interaction between the defects is mediated by a partially occupied spin-polarized defect band. However, this mechanism requires considerable fine-tuning: The defect states need to be sufficiently extended to mediate the FM coupling via Zener's double exchange, yet the band width must be small enough for the Stoner criterion to be satisfied [10-18]. Consequently, theoretical results reported so far are strongly method dependent: In LDA or generalized gradient approximation (GGA) calculations, the $2 p$ defect state tends to come out too extended, and hence their predictions of ferromagnetism might be overly optimistic. On the other hand, calculations using LDA plus Hubbard $U$ [16] or a self-interaction correction [17,18] emphasize Coulomb correlation in the $2 p$ hole states, and favor a splitting of the partially occupied impurity levels. The Coulomb correlation strongly localizes the $2 p$ hole states (and the associated magnetic moments) and thus, in combination with a local Jahn-Teller distortion, impedes ferromagnetism [16-19].

In this Letter, we study impurity pairs that are expected to play a significant role at the high impurity concentrations typically used. We demonstrate that $\mathrm{C}$ or $\mathrm{N}$ impurities may interact attractively in both $\mathrm{MgO}$ and $\mathrm{ZnO}$. In contrast to the earlier predictions of a metallic impurity band, we find that $\mathrm{MgO}_{1-x} \mathrm{~N}_{x}$, after pairing, becomes an insulator with nonmagnetic or antiferromagnetically coupled impurity molecules. Next, we address the magnetic properties of the open-shell "defect molecule" $\mathrm{C}_{2}$ in these oxides hosts. Our calculations suggest that the $d^{0}$ magnetism observed in the $\mathrm{C}$-doped $\mathrm{ZnO}$ may well be due to a magnetic interaction between these species mediated by conduction band electrons.

We performed LDA calculations for the $\mathrm{N}$ - or C-doped $\mathrm{MgO}$, using fully relaxed structures with two substitutional impurity atoms in a $2 \times 2 \times 2$ supercell. The full-potential augmented plane wave plus local orbital code WIEN2K was used [20]. The muffin-tin spheres were chosen to be 2.1 Bohr for $\mathrm{Mg}$ and 1.3 for $\mathrm{O}, \mathrm{N}$, and $\mathrm{C}$, the plane-wave cutoff of 15 Ry for the interstitial wave functions, and a $5 \times 5 \times 5$ k-point mesh for integration over the Brillouin zone. The Kohn-Sham band gap of bulk $\mathrm{MgO}$ is calculated to be $5.0 \mathrm{eV}$ by LDA, much smaller than the experimental value of $7.8 \mathrm{eV}$. We note that the impurity bands of concern are all located inside the (too small) LDA gap (see Figs. 1 and 2). Thus, the results can be interpreted in a physically meaningful way, despite the obvious shortcomings of LDA. Note also that our calculations using GGA gave almost the same results as LDA.

We start with the calculations of two substitutional $\mathrm{N}$ atoms at various oxygen sites in a $\mathrm{Mg}_{32} \mathrm{O}_{30} \mathrm{~N}_{2}$ supercell, 


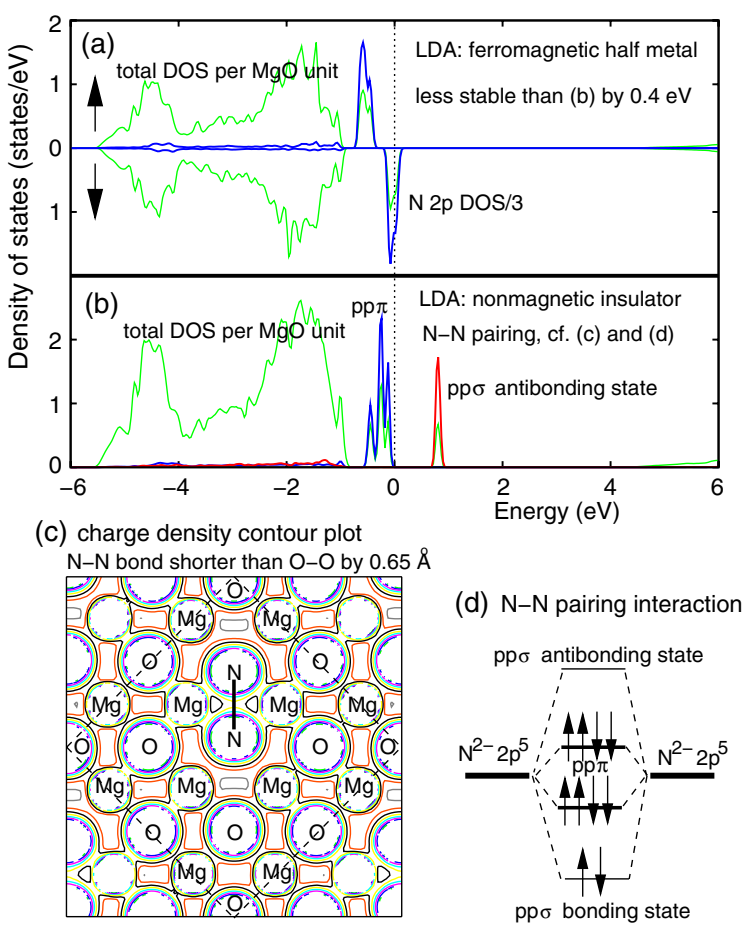

FIG. 1 (color online). Total and N $2 p$ DOS for two N atoms with the largest (a) or smallest (b) possible separation, in a $\mathrm{Mg}_{32} \mathrm{O}_{30} \mathrm{~N}_{2}$ supercell. The Fermi level is set to zero. (c) Charge density contour plot for case (b) $\left(0.1-0.8 e / \AA^{3}\right)$. The dashed square marks the supercell in the $a b$ plane. (d) Schematic energy level diagram of a N-N pair. Except for the $p p \sigma$ antibonding state, all levels are fully occupied, consistent with (b).

for a comparison with the literature. Figure 1(a) shows the density of states (DOS) obtained with the longest N-N distance of $7.29 \AA$. It is a FM half-metal (FMHM) as reported in the literature $[4,15]$. This is due to the $2 / 3$ filled down-spin N $2 p$ impurity band split off from the fully occupied up-spin band via Hund exchange. After placing the second $\mathrm{N}$ atom at the nearest-neighbor site, the total energy is lowered by $0.4 \mathrm{eV}$ compared to the well separated $\mathrm{N}$ impurities [Fig. 1(a)] and the N-N distance becomes $0.65 \AA$ shorter than the O-O distance [Fig. 1(c)]. Figure 1(d) shows a schematic level diagram of the N-N pairing interaction. The $p p \sigma$ bonding state and the $p p \pi$ states can accommodate all of the ten electrons, and the $p p \sigma$ antibonding state is empty; thus the pairing leads to a nonmagnetic insulating state, see also Fig. 1(b).

Now we turn to the C-doped $\mathrm{MgO}$. For the $\mathrm{Mg}_{32} \mathrm{O}_{30} \mathrm{C}_{2}$ supercell with the longest C-C distance, our LDA calculation gives the same FMHM solution (due to the $1 / 3$ filled C- $2 p$ down-spin impurity band, not shown here) as reported in the literature [4]. However, the pairing interaction for $\mathrm{C}$ in $\mathrm{MgO}$ is even more significant than for $\mathrm{N}$ : The $\mathrm{C}-\mathrm{C}$ distance is $1.47 \AA$, being only half of the O-O distance of $2.97 \AA$, see Fig. 2(b). The corresponding bonding energy gain is large, being $3.2 \mathrm{eV}$ compared to well-separated

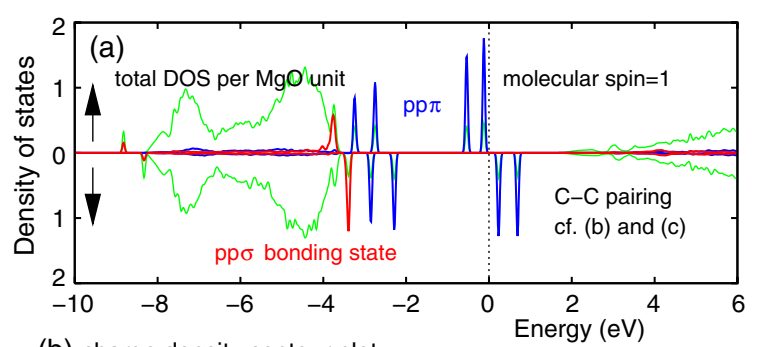

(b) charge density contour plot

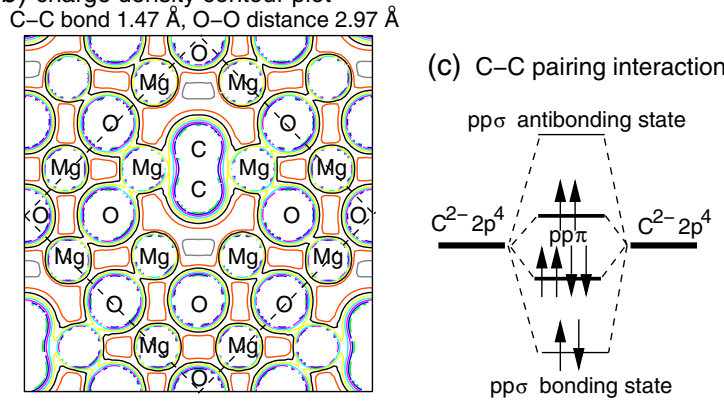

FIG. 2 (color online). (a) Total and C $2 p$ DOS for two nearestneighbor $\mathrm{C}$ impurities in a $\mathrm{Mg}_{32} \mathrm{O}_{30} \mathrm{C}_{2}$ supercell. It is an insulator with a molecular spin $=1$. (b) Charge density contour plot (0.1-0.8 e/ $\left.\AA^{3}\right)$. (c) Schematic energy level diagram of a C-C molecular ion, isoelectronic to an $\mathrm{O}_{2}$ molecule. The nearly degenerate $p p \pi$ antibonding state is half-filled and is in a spin triplet, consistent with the DOS in (a).

$\mathrm{C}$ impurities. This much stronger $\mathrm{C}-\mathrm{C}$ pairing effect can be understood by noticing that there are two electrons less in the $p p \pi$ antibonding level of a C-C pair than in $\mathrm{N}-\mathrm{N}$, see Figs. 2(c) and 1(d). It is not surprising that the $\mathrm{C}-\mathrm{C}$ pair has a molecular spin $=1$, because it is isoelectronic to the free $\mathrm{O}_{2}$ molecule, the spin triplet ground state of which is well known. Similarly, a molecular spin was found in $\mathrm{N}_{2}^{2-}$ [21] and $\mathrm{O}_{2}^{-}[22,23]$ molecular ions being part of ionic compounds. As seen in Fig. 2(a), the C-C dimer has a huge $p p \sigma$ bonding-antibonding splitting of more than $6 \mathrm{eV}$, as well as a sizable $p p \pi$ bonding-antibonding splitting of $3 \mathrm{eV}$. It is important to note that the formal $p p \pi$ doublet has a crystal field splitting of $0.4 \mathrm{eV}$ due to atomic relaxations, but this is still smaller than the exchange splitting of $0.8 \mathrm{eV}$. As a result, the $\mathrm{C}_{2}$ molecular spin $=1$ is preserved in the calculations, which show that indeed the spin triplet is by $0.19 \mathrm{eV}$ more stable than a spin singlet. Moreover, there is a small gap of about $0.4 \mathrm{eV}$ between the spin-up and down $p p \pi$ antibonding states. Thus, an antiferromagnetic (AFM) superexchange is expected between those spin $=1 \mathrm{C}_{2}$ molecules, and our calculations show that the AFM coupling between two most distant $\mathrm{C}_{2}$ molecules in a $\mathrm{Mg}_{32} \mathrm{O}_{28} \mathrm{C}_{4}$ supercell is more stable than the FM by $20 \mathrm{meV}$. (We note that this value would be somewhat reduced if in the calculations the true band gap rather than the smaller LDA gap and a larger supercell were used). Such an AFM coupling was also found in the molecular magnets $\mathrm{SrN}$ [21] and $\mathrm{Rb}_{4} \mathrm{O}_{6}$ [23]. In other 


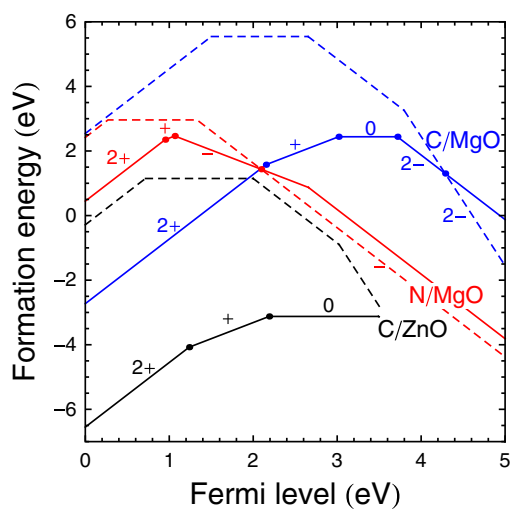

FIG. 3 (color online). Formation energy of $\mathrm{C}$ and $\mathrm{N}$ substitutional impurities at $\mathrm{O}$ sites in $\mathrm{MgO}$ and $\mathrm{ZnO}$ for $t w o$ isolated single impurities (dashed lines) and one impurity-pair (full lines) in different charge states with respect to thermodynamic reservoirs of atomic $\mathrm{C}$ and $\mathrm{N}$, and gas-phase $\mathrm{O}_{2}$. The Fermi level is measured relative to the valence band maximum.

words, our calculations demonstrate that substitutional-Cdoped $\mathrm{MgO}$, in the absence of other defects, is expected to be an insulator, and has impurities with a molecular spin $=1$ and finite AFM coupling. Thus, the present results are in strong contrast to previous work assuming single impurities, predicting a FMHM by LDA [4] or an insulator with a local atomic spin $=1$ by self-interaction correction $[17,18]$.

Next, we address the question of whether the presence of intrinsic defects or codoping could possibly change the position of the Fermi level $E_{F}$ and thus induce magnetism. Formation energies of single impurities and impurity pairs in various charge states were calculated in the presence of a compensating background charge [24], using a 128-atom supercell for $\mathrm{MgO}$. An electrostatic correction for the finite supercell size was included [25]. These GGA calculations were performed using the projector-augmented-wave method [26] as implemented in the VASP code [27]. The results shown in Fig. 3 confirm our negative conclusions regarding magnetism in $\mathrm{MgO}$ : Single impurities are energetically favorable only in $n$-type $\mathrm{MgO}$, but the resulting $\mathrm{C}^{2-}$ and $\mathrm{N}^{-}$species are closed shell and hence nonmagnetic. In $p$-type $\mathrm{MgO}$ (low $E_{F}$ values) we find that the formation of single impurities is energetically very costly, and pairing is exothermic. The thermodynamically stable species (cf. Fig. 3) are $\mathrm{N}_{2}^{2+}$ and $\mathrm{N}_{2}^{-}$, carrying a molecular spin $=1$ and $=\frac{1}{2}$, respectively. Notably the $\mathrm{N}_{2}^{2+}$ becomes more stable and magnetic by loosing two antibonding spindown $p p \pi$ electrons in $p$-type $\mathrm{MgO}$, and the $\mathrm{N}-\mathrm{N}$ distance is reduced to $1.29 \AA$. Being isoelectronic to neutral $\mathrm{C}_{2}$ (cf. Fig. 2), the insulating band structure of $\mathrm{N}_{2}^{2+} / \mathrm{MgO}$ (not shown here) results in an AFM superexchange, as discussed above. For $\mathrm{C} / \mathrm{MgO}$, the stable $\mathrm{C}_{2}^{2+}, \mathrm{C}_{2}^{+}$and $\mathrm{C}_{2}^{0}$ species (cf. Figure 3) carry a molecular spin $=0,=\frac{1}{2}$ and $=1$, respectively, but again AFM coupling (if any) is expected due to the samples being band insulators. Thus,

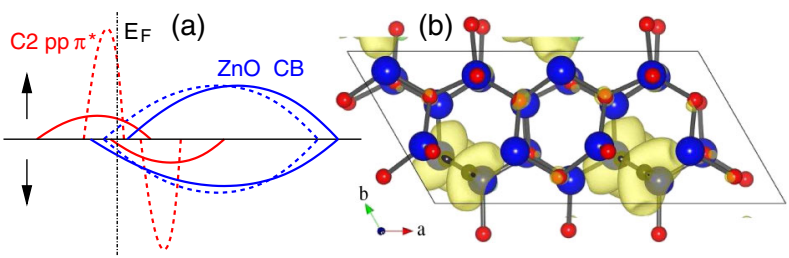

FIG. 4 (color online). (a) Resonance of the spin-split $\mathrm{C}_{2}$ impurity levels with the conduction band bottom of $\mathrm{ZnO}$ stabilizes FM order. Solid (dashed) DOS curves standing for the switch-on (off) hybridization illustrate the energy lowering. (b) Top view into the $a b$ plane of the spin-density isosurface of the C-doped $\mathrm{ZnO}$ in the FM ground state. Big blue (small red) spheres stand for $\mathrm{Zn}(\mathrm{O})$ atoms. The spin density spreads over the $\mathrm{C}_{2}$ dimer and the neighboring $\mathrm{Zn}$ atoms.

the route to $d^{0}$ magnetism envisaged previously, namely, via $2 p$ holes in an impurity band formed from diluted impurities [4-6,10-15], appears not well founded, as isolated impurities are far from being thermodynamically stable. The paired state, on the other hand, is insulating and hence does not support FM interactions.

In view of the above spin $=1 \mathrm{C}_{2}$ molecule, we wish to find a suitable oxide semiconductor which, after $\mathrm{C}$ doping, becomes FM. This requires that its band gap should be about 3-4 eV, with the bottom of its conduction band overlapping the spin-polarized antibonding $p p \pi$ impurity state [see Fig. 2(a)]. Then their level resonance [see Fig. 4(a)] will make the bottom of the conduction band spin polarized and result in a long-range FM [28]. As such, we propose $\mathrm{ZnO}$ as a candidate, having a band gap of $3.4 \mathrm{eV}$. First, we calculate the formation energies of isolated and paired $\mathrm{C}$ in $\mathrm{ZnO}$ in a 108-atom supercell with GGA, including an effective Hubbard $U=9 \mathrm{eV}$ for the $\mathrm{Zn} 3 d$ electrons to correct the underbinding of the $3 d$ states. Again, we find pairing to be preferred (cf. Fig. 3) as the Fermi level runs through the whole band gap. In order to explore the consequence of impurity-pair levels resonating with the host conduction band, we used the screened hybrid functional of the Heyd-Scuseria-Ernzerhof (HSE) type $[29,30]$, which improves the description of semiconductors and their defect states [31-33] by mixing in a small amount (denoted by $\alpha$ ) of the Hartree-Fock exchange to the DFT exchange. In improvement over previous LDA and GGA studies [6,11,12], HSE yields, for $\mathrm{ZnO}$, a band gap in excellent agreement with the experimental value when $\alpha=0.375$ is used [33]. We have studied the specific case of two $\mathrm{C}-\mathrm{C}$ dimers under the condition of charge neutrality, using the VASP code and a $4 \times 2 \times 2$ $\left(\mathrm{Zn}_{32} \mathrm{O}_{28} \mathrm{C}_{4}\right)$ supercell together with a $2 \times 4 \times 4 \mathbf{k}$-point grid. The plane-wave cutoff energy of $400 \mathrm{eV}$ has been set, and $\alpha=0.375$ and the screening factor of $0.2 \AA^{-1}$ have been used. After relaxation of a nearest-neighbor impurity pair, we obtain a C-C dimer placed asymmetrically in the $\mathrm{ZnO}$ matrix: one of the oxygen vacancies is occupied by the dimer, while the other vacancy shrinks as the adjacent 
Zn atoms relax inward. The C-C bond length is $1.31 \AA$, even shorter than for the $\mathrm{C}-\mathrm{C}$ dimer in $\mathrm{MgO}$ matrix. The two $\mathrm{C}_{2}$ dimers are separated by $6.5 \AA$. The $\mathrm{C}_{2}$ molecule in a single vacancy hybridizes considerably with the neighboring $\mathrm{Zn}$ atoms. Hence, the antibonding molecular states broaden into resonances with the conduction band and the spin density spreads over the $\mathrm{C}_{2}$ dimers and the neighboring $\mathrm{Zn}$ atoms, see Fig. 4(b). The spin moment is calculated to be $1.4 \mu_{B}$ per $C_{2}$ dimer (reduced from its formal value of $2 \mu_{B}$ ) due to the hybridization and partial delocalization. It is most important to note that after a full relaxation of the supercell having a FM or AFM coupling between the two $\mathrm{C}_{2}$ dimers, the FM state turns out by our HSE calculations to be more stable than the AFM state by $36 \mathrm{meV}$, indicating that $\mathrm{C}$-doped $\mathrm{ZnO}$ may indeed be a $d^{0}$ FM semiconductor.

To conclude, we demonstrated by LDA calculations that $\mathrm{C}$ or $\mathrm{N}$ substitutional impurities in $p$-type $\mathrm{MgO}$ prefer pairing, which renders $\mathrm{N}$-doped $\mathrm{MgO}$ a spin $=1$ antiferromagnetic ( $\operatorname{spin}=0$ nonmagnetic) insulator with the $2+$ charged (neutral) $\mathrm{N}-\mathrm{N}$ pairs, and C-doped $\mathrm{MgO}$ an insulating molecular antiferromagnet. Moreover, we find that $\mathrm{C}$-doped $\mathrm{ZnO}$ could well be a $d^{0}$ ferromagnetic semiconductor due to a subtle interaction between the spin $=1$ $\mathrm{C}_{2} p p \pi$ antibonding state and the close-lying bottom of the host conduction band, as supported by hybrid functional calculations. We thus propose a mechanism-impurity pairing and molecular magnetism in resonance with the host band or not- to determine if a $d^{0}$ magnetism could be present in $\mathrm{C}$ or $\mathrm{N}$-doped oxides. Obviously, this mechanism applies also to other open-shell impurities than those discussed in the present Letter. We trust that the nature of the defect complexes giving rise to magnetism in $\mathrm{MgO}$ and $\mathrm{ZnO}$ will soon be identified by experiments.

This study is supported by Deutsche Forschungsgemeinschaft via SFB 608 (H.W.) and SFB 491 (S.S. and P. K.). A. S. and S.P. greatly acknowledge CASPUR supercomputing center for assistance in performing the hybrid functional calculations on its HPC clusters.

*Corresponding author: wu@ph2.uni-koeln.de

[1] J. M. D. Coey, Solid State Sci. 7, 660 (2005).

[2] G. Bouzerar and T. Ziman, Phys. Rev. Lett. 96, 207602 (2006).

[3] A. Zunger, S. Lany, and H. Raebiger, Physics 3, 53 (2010).

[4] K. Kenmochi, M. Seike, K. Sato, A. Yanase, and H. Katayama-Yoshida, Jpn. J. Appl. Phys. 43, L934 (2004); K. Kenmochi, V. A. Dinh, K. Sato, A. Yanase, and H. Katayama-Yoshida, J. Phys. Soc. Jpn. 73, 2952 (2004).

[5] I. S. Elfimov, A. Rusydi, S. I. Csiszar, Z. Hu, H. H. Hsieh, H.-J. Lin, C. T. Chen, R. Liang, and G. A. Sawatzky, Phys. Rev. Lett. 98, 137202 (2007).

[6] H. Pan, J. B. Yi, L. Shen, R. Q. Wu, J. H. Yang, J. Y. Lin, Y. P. Feng, J. Ding, L. H. Van, and J. H. Yin, Phys. Rev. Lett. 99, 127201 (2007).
[7] X. J. Ye, H. A. Song, W. Zhong, M. H. Xu, X. S. Qi, C. Q. Jin, Z. X. Yang, C. T. Au, and Y. W. Du, J. Phys. D: Appl. Phys. 41, 155005 (2008).

[8] S. Zhou, Q. Xu, K. Potzger, G. Talut, R. Grötzschel, J. Fassbender, M. Vinnichenko, J. Grenzer, M. Helm, H. Hochmuth, M. Lorenz, M. Grundmann, and H. Schmidt, Appl. Phys. Lett. 93, 232507 (2008).

[9] T. S. Herng, S. P. Lau, L. Wang, B. C. Zhao, S. F. Yu, M. Tanemura, A. Akaike, and K. S. Teng, Appl. Phys. Lett. 95, 012505 (2009).

[10] L. Shen, R. Q. Wu, H. Pan, G. W. Peng, M. Yang, Z. D. Sha, and Y. P. Feng, Phys. Rev. B 78, 073306 (2008).

[11] Haowei Peng, H. J. Xiang, S.-H. Wei, Shu-Shen Li, JianBai Xia, and Jingbo Li, Phys. Rev. Lett. 102, 017201 (2009).

[12] Xiangyang Peng and Rajeev Ahuja, Appl. Phys. Lett. 94, 102504 (2009).

[13] Q. Wang, Q. Sun, and P. Jena, New J. Phys. 11, 063035 (2009).

[14] S. W. Fan, K. L. Yao, and Z. L. Liu, Appl. Phys. Lett. 94, 152506 (2009).

[15] P. Mavropoulos, M. Ležaić, and S. Blügel, Phys. Rev. B 80, 184403 (2009).

[16] V. Pardo and W.E. Pickett, Phys. Rev. B 78, 134427 (2008).

[17] A. Droghetti, C. D. Pemmaraju, and S. Sanvito, Phys. Rev. B 78, 140404(R) (2008).

[18] A. Droghetti and S. Sanvito, Appl. Phys. Lett. 94, 252505 (2009).

[19] J. A. Chan, S. Lany, and A. Zunger, Phys. Rev. Lett. 103, 016404 (2009).

[20] P. Blaha, K. Schwarz, G. Madsen, D. Kvasnicka, and J. Luitz, WIEN2K, (2001), ISBN 3-9501031-1-2.

[21] O. Volnianska and P. Boguslawski, Phys. Rev. B 77, 220403(R) (2008).

[22] J. J. Attema, G. A. de Wijs, G. R. Blake, and R. A. de Groot, J. Am. Chem. Soc. 127, 16325 (2005).

[23] J. Winterlik, G. H. Fecher, C. A. Jenkins, C. Felser, C. Mühle, K. Doll, M. Jansen, L. M. Sandratskii, and J. Kübler, Phys. Rev. Lett. 102, 016401 (2009).

[24] C. G. Van de Walle and J. Neugebauer, J. Appl. Phys. 95, 3851 (2004), and references therein.

[25] G. Makov and M.C. Payne, Phys. Rev. B 51, 4014 (1995).

[26] P. E. Blöchl, Phys. Rev. B 50, 17953 (1994).

[27] G. Kresse and J. Furthmüller, Comput. Mater. Sci. 6, 15 (1996).

[28] S. Lany, H. Raebiger, and A. Zunger, Phys. Rev. B 77, 241201(R) (2008); H. Raebiger, S. Lany, and A. Zunger, Phys. Rev. Lett. 101, 027203 (2008).

[29] J. Heyd, G. E. Scuseria, and M. Ernzerhof, J. Chem. Phys. 118, 8207 (2003).

[30] B. G. Janesko, T. M. Henderson, and G. E. Scuseria, Phys. Chem. Chem. Phys. 11, 443 (2009).

[31] M. Marsman, J. Paier, A. Stroppa, and G. Kresse, J. Phys. Condens. Matter 20, 064201 (2008).

[32] A. Stroppa and G. Kresse, Phys. Rev. B 79, 201201(R) (2009).

[33] F. Oba, A. Togo, I. Tanaka, J. Paier, and G. Kresse, Phys. Rev. B 77, 245202 (2008). 